\title{
RECURRENCE COEFFICIENTS OF GENERALIZED CHARLIER POLYNOMIALS AND THE FIFTH PAINLEVÉ EQUATION
}

\author{
GALINA FILIPUK AND WALTER VAN ASSCHE \\ (Communicated by Sergei K. Suslov)
}

\begin{abstract}
We investigate generalizations of the Charlier polynomials on the lattice $\mathbb{N}$, on the shifted lattice $\mathbb{N}+1-\beta$, and on the bi-lattice $\mathbb{N} \cup$ $(\mathbb{N}+1-\beta)$. We show that the coefficients of the three-term recurrence relation for the orthogonal polynomials are related to solutions of the fifth Painlevé equation $\mathrm{P}_{\mathrm{V}}$ (which can be transformed to the third Painlevé equation). Initial conditions for different lattices can be transformed to the classical solutions of $\mathrm{P}_{\mathrm{V}}$ with special values of the parameters.
\end{abstract}

\section{INTRODUCTION}

In this paper we investigate the recurrence coefficients of orthogonal polynomials, in particular generalized Charlier polynomials, and show that they are related to special solutions of a continuous Painlevé equation when viewed as functions of one of the parameters. We are interested to see how the properties of the orthogonal polynomials are related to properties of transformations of the Painlevé equation.

The paper is organized as follows. In the introduction we shall first review orthogonal polynomials for a generalized Charlier weight on different lattices and their main properties, following [14. Next we shall briefly recall the fifth Painlevé equation and its Bäcklund transformations. We then derive the Toda system and show that the recurrence coefficients can be expressed in terms of the solutions of the fifth Painlevé equation. Finally we study the initial conditions of the recurrence coefficients for different lattices.

1.1. Orthogonal polynomials for the generalized Charlier weight. One of the most important properties of orthogonal polynomials is a three-term recurrence relation. For a sequence $\left(p_{n}(x)\right)_{n \in \mathbb{N}}$ of orthonormal polynomials for a weight $w$ on the lattice $\mathbb{N}=\{0,1,2,3, \ldots\}$,

$$
\sum_{k=0}^{\infty} p_{n}(k) p_{m}(k) w(k)=\delta_{n, m},
$$

Received by the editors June 15, 2011 and, in revised form, June 30, 2011.

2010 Mathematics Subject Classification. Primary 34M55, 33E17; Secondary 33C47, 42C05, 65Q30.

Key words and phrases. Orthogonal polynomials, recurrence coefficients, Painlevé equations, Bäcklund transformations, classical solutions.

The first author is partially supported by Polish MNiSzW Grant N N201 397937.

The second author was supported by the Belgian Interuniversity Attraction Pole P6/02, FWO Grant G.0427.09 and KU Leuven Research Grant OT/08/033.

(C) 2012 American Mathematical Society Reverts to public domain 28 years from publication 
where $\delta_{n, m}$ is the Kronecker delta, this relation takes the following form:

$$
x p_{n}(x)=a_{n+1} p_{n+1}(x)+b_{n} p_{n}(x)+a_{n} p_{n-1}(x) .
$$

For the monic polynomials $P_{n}$ related to orthonormal polynomials $p_{n}(x)=\gamma_{n} x^{n}+$ ... with

$$
\frac{1}{\gamma_{n}^{2}}=\sum_{k=0}^{\infty} P_{n}^{2}(k) w(k),
$$

the recurrence relation is given by

$$
x P_{n}(x)=P_{n+1}(x)+b_{n} P_{n}(x)+a_{n}^{2} P_{n-1}(x) .
$$

The (classical) Charlier polynomials (4, Chapter VI], [14) are orthogonal on the lattice $\mathbb{N}$ with respect to the Poisson distribution:

$$
\sum_{k=0}^{\infty} C_{n}(k ; a) C_{m}(k ; a) \frac{a^{k}}{k !}=a^{-n} \exp (a) n ! \delta_{n, m}, \quad a>0 .
$$

The weight $w_{k}=w(k)=a^{k} / k$ ! satisfies the Pearson equation

$$
\nabla w(x)=\left(1-\frac{x}{a}\right) w(x),
$$

where $\nabla$ is the backward difference operator

$$
\nabla f(x)=f(x)-f(x-1) .
$$

Here the function $w(x)=a^{x} / \Gamma(x+1)$ gives the weights $w_{k}=w(k)=a^{k} / k$ !. The Pearson equation for the Charlier polynomials is, hence, of the form

$$
\nabla[\sigma(x) w(x)]=\tau(x) w(x)
$$

with $\sigma=1$ and $\tau$ a polynomial of degree 1 .

Recall that the classical orthogonal polynomials are characterized by the Pearson equation (3) with $\sigma$ a polynomial of degree at most 2 and $\tau$ a polynomial of degree 1. Note that in (3) the operator $\nabla$ is used for orthogonal polynomials on the lattice and it is replaced by differentiation in the case of orthogonal polynomials on an interval of the real line. The Pearson equation plays an important role in the theory of classical orthogonal polynomials since it allows us to find many useful properties of these polynomials. It is known that the recurrence coefficients of the Charlier polynomials are given explicitly by $a_{n}^{2}=n a$ and $b_{n}=n+a$ for $n \in \mathbb{N}$.

The Charlier weight can be generalized [14] with one additional parameter. One can use the weight function

$$
w(x)=\frac{\Gamma(\beta) a^{x}}{\Gamma(\beta+x) \Gamma(x+1)},
$$

which gives the weight

$$
w_{k}=w(k)=\frac{a^{k}}{(\beta)_{k} k !}, \quad a>0, \beta>0,
$$

on the lattice $\mathbb{N}$. The case $\beta=1$ was first considered in 9 and later analyzed in [17. The Pearson equation is now of the form

$$
\nabla w(x)=\frac{a-x(\beta-1)-x^{2}}{a} w(x) .
$$


The monic orthogonal polynomials $P_{n}(x ; a, \beta)$ for the weight (4) satisfy

$$
\sum_{k=0}^{\infty} P_{n}(k ; a, \beta) P_{m}(k ; a, \beta) \frac{a^{k}}{(\beta)_{k} k !}=0, \quad n \neq m,
$$

and the three-term recurrence relation is

$$
x P_{n}(x ; a, \beta)=P_{n+1}(x ; a, \beta)+b_{n} P_{n}(x ; a, \beta)+a_{n}^{2} P_{n-1}(x ; a, \beta),
$$

with initial conditions $P_{-1}=0$ and $P_{0}=1$. If we replace $a$ by $\beta a$ and then take the limit $\beta \rightarrow \infty$, we recover the Charlier polynomials 14 .

Theorem 1.1 ([14, Th. 2.1]). The recurrence coefficients for the orthogonal polynomials defined by (5) for the weight (4) on the lattice $\mathbb{N}$ satisfy

$$
\begin{aligned}
& b_{n}+b_{n-1}-n+\beta=\frac{a n}{a_{n}^{2}}, \\
& \left(a_{n+1}^{2}-a\right)\left(a_{n}^{2}-a\right)=a\left(b_{n}-n\right)\left(b_{n}-n+\beta-1\right),
\end{aligned}
$$

with initial conditions

$$
a_{0}^{2}=0, \quad b_{0}=\frac{\sqrt{a} I_{\beta}(2 \sqrt{a})}{I_{\beta-1}(2 \sqrt{a})},
$$

where $I_{\beta}$ is the modified Bessel function.

The system (6) - (7) can be identified as a limiting case of an asymmetric discrete Painlevé equation. In this paper we show that it can be obtained from the Bäcklund transformation of the fifth Painlevé equation.

One can use the weight (4) on the shifted lattice $\mathbb{N}+1-\beta$ when $\beta<2$ and one can also combine both lattices when $0<\beta<2$ to obtain the bi-lattice $\mathbb{N} \cup(\mathbb{N}+1-\beta)$. The orthogonality measure for the bi-lattice is a linear combination of the measures on $\mathbb{N}$ and $\mathbb{N}+1-\beta$. The weight $w$ in (44) on the shifted lattice $\mathbb{N}+1-\beta=$ $\{1-\beta, 2-\beta, 3-\beta, \ldots\}$ is given by

$$
v_{k}:=w(k+1-\beta)=\frac{\Gamma(\beta) a^{1-\beta}}{\Gamma(2-\beta)} \frac{a^{k}}{k !(2-\beta)_{k}}, \quad k \in \mathbb{N} .
$$

We see that up to a constant factor it is equal to the weight on the original lattice $\mathbb{N}$ with different parameters. The corresponding monic orthogonal polynomials $Q_{n}$ on the shifted lattice satisfy

$$
\sum_{k=0}^{\infty} Q_{n}(k+1-\beta) Q_{m}(k+1-\beta) v_{k}=0, \quad n \neq m,
$$

and one has

$$
Q_{n}(x)=P_{n}(x+\beta-1 ; a, 2-\beta) .
$$

In [14, Th. 2.2] it is shown that the recurrence coefficients in the three-term recurrence relation

$$
x Q_{n}(x)=Q_{n+1}(x)+\hat{b}_{n} Q_{n}(x)+\hat{a}_{n}^{2} Q_{n-1}(x)
$$

satisfy the same system (6)-(7) (with hats) but with initial conditions

$$
\hat{a}_{0}^{2}=0, \quad \hat{b}_{0}=\frac{\sqrt{a} I_{-\beta}(2 \sqrt{a})}{I_{1-\beta}(2 \sqrt{a})} .
$$

Using the orthogonality measure $\mu=c_{1} \mu_{1}+c_{2} \mu_{2}$, where $c_{1}, c_{2}>0, \mu_{1}$ is the discrete measure on $\mathbb{N}$ with weights $w_{k}=w(k)$ and $\mu_{2}$ is the discrete measure on $\mathbb{N}+1-\beta$ with weights $v_{k}=w(k+1-\beta)$, one can study the monic orthogonal 
polynomials $R_{n}(x)=R_{n}(x ; a, \beta, \tau)$, where $\tau=c_{2} / c_{1}>0$, satisfying the three-term recurrence relation

$$
x R_{n}(x)=R_{n+1}(x)+\tilde{b}_{n} R_{n}(x)+\tilde{a}_{n}^{2} R_{n-1}(x) .
$$

The orthogonality relation is given by

$$
c_{1} \sum_{k=0}^{\infty} R_{n}(k) R_{m}(k) w_{k}+c_{2} \sum_{k=0}^{\infty} R_{n}(k+1-\beta) R_{m}(k+1-\beta) v_{k}=0, \quad m \neq n .
$$

According to [14, Th. 2.3] the recurrence coefficients $\tilde{a}_{n}^{2}$ and $\tilde{b}_{n}$ satisfy the system (6) - (7) (with tilde) but with initial conditions

$$
\tilde{a}_{0}^{2}=0, \quad \tilde{b}_{0}=\sqrt{a} \frac{I_{\beta}(2 \sqrt{a})+\tau I_{-\beta}(2 \sqrt{a})}{I_{\beta-1}(2 \sqrt{a})+\tau I_{1-\beta}(2 \sqrt{a})} .
$$

Thus, it is shown in 14 that the orthogonal polynomials for the generalized Charlier weight on the lattice $\mathbb{N}$, on the shifted lattice $\mathbb{N}+1-\beta$ and on the bilattice $\mathbb{N} \cup(\mathbb{N}+1-\beta)$ have recurrence coefficients $a_{n}^{2}$ and $b_{n}$ which satisfy the same nonlinear system of discrete (recurrence) equations but the initial conditions are different for each case.

1.2. The fifth Painlevé equation and its Bäcklund transformations. The Painlevé equations possess the so-called Painlevé property: the only movable singularities of the solutions are poles. They are often referred to as nonlinear special functions and have numerous applications in mathematics and mathematical physics. There is a vast bibliography related to various aspects of the Painlevé equations, their Bäcklund transformations and special solutions (see, e.g., 7], 8], [13. Ch. 32 and p. 740], and [15] in particular for $\mathrm{P}_{\mathrm{V}}$ ). We will mainly use the notation from the book of Gromak et al. 8] and include only those references that we need for our analysis.

The fifth Painlevé equation $\mathrm{P}_{\mathrm{V}}$ is given by

$$
y^{\prime \prime}=\left(\frac{1}{2 y}+\frac{1}{y-1}\right)\left(y^{\prime}\right)^{2}-\frac{y^{\prime}}{t}+\frac{(y-1)^{2}}{t^{2}}\left(A y+\frac{B}{y}\right)+\frac{C y}{t}+\frac{D y(y+1)}{y-1},
$$

where $y=y(t)$ and $A, B, C, D$ are arbitrary complex parameters.

The fifth Painlevé equation $\mathrm{P}_{\mathrm{V}}$ in case $C \neq 0, D=0$ can be reduced to the third Painlevé equation. There exists a Bäcklund transformation between solutions of the fifth Painlevé equation with $D=0$.

Theorem $1.2([2,16])$. Let $y=y(t)$ be a solution of $P_{\mathrm{V}}$ with parameters $A, B, C$, $D$ given by $B=-p^{2} / 2 \neq 0, C \neq 0$ and $D=0$. Then the function $y_{1}=y_{1}(t)$ given by

$$
y_{1}=1-\frac{2 C t y^{2}(y-1)}{2 A y^{2}(y-1)^{2}-\left(p(y-1)-t y^{\prime}\right)^{2}}
$$

is a solution of $P_{\mathrm{V}}$ with parameters $A_{1}=A, B_{1}=-(p-1)^{2} / 2, C_{1}=C, D_{1}=0$. The inverse transformation is given by

$$
y=1-\frac{2 C t y_{1}^{2}\left(y_{1}-1\right)}{2 A y_{1}^{2}\left(y_{1}-1\right)^{2}-\left((1-p)\left(y_{1}-1\right)-t y_{1}^{\prime}\right)^{2}} .
$$


In case $D \neq 0$ there exists another well-known transformation.

Theorem 1.3 ([8, Th. 39.1], [13, $§ 32.7(\mathrm{v})]$, [7]). If $y=y(t)$ is the solution of the fifth Painlevé equation (12) with parameters $A, B, C, D$, then the transformation

$$
T_{\varepsilon_{1}, \varepsilon_{2}, \varepsilon_{3}}: y \rightarrow y_{1}
$$

gives another solution $y_{1}=y_{1}(t)$ with new values of the parameters $A_{1}, B_{1}, C_{1}, D_{1}$, where

$$
\begin{aligned}
& y_{1}=1-\frac{2 d t y}{t y^{\prime}-a y^{2}+(a-b+d t) y+b}, \\
& A_{1}=-\frac{1}{16 D}(C+d(1-a-b))^{2}, \quad B_{1}=\frac{1}{16 D}(C-d(1-a-b))^{2}, \\
& C_{1}=d(b-a), \quad D_{1}=D, \\
& a=\varepsilon_{1} \sqrt{2 A}, \quad b=\varepsilon_{2} \sqrt{-2 B}, \quad d=\varepsilon_{3} \sqrt{-2 D}, \quad \varepsilon_{j}^{2}=1, \quad j \in\{1,2,3\} .
\end{aligned}
$$

Example. Let $y=y_{n}(t)$ be a solution of $\mathrm{P}_{\mathrm{V}}$ with parameters $A=-B=$ $n^{2} / 8, C=0, \quad D=-8$. Then the function

$$
y_{n+1}=1-\frac{16 t \varepsilon y}{2 t y^{\prime}+n y^{2}+8 t \varepsilon y-n}
$$

is a solution of $\mathrm{P}_{\mathrm{V}}$ with $A=-B=(n+1)^{2} / 8, \quad C=0, \quad D=-8, \quad \varepsilon^{2}=1$.

Remark 1. For our purposes it is sufficient to use the standard Bäcklund transformations of the Painlevé transcendents which are given in the NIST Digital Library of Mathematical Functions (DLMF project) 1] see [13, Chapter 32]. This also contains various algebraic aspects of the Painlevé equations. The interested reader can easily reformulate the transformations within the framework of Noumi-Yamada's birational representation of the affine Weyl groups [12].

1.3. Relation to the third Painlevé equation. The third Painlevé equation $\mathrm{P}_{\mathrm{III}}$ is given by

$$
u^{\prime \prime}=\frac{u^{\prime 2}}{u}-\frac{u^{\prime}}{z}+\frac{1}{z}\left(\tilde{\alpha} u^{2}+\tilde{\beta}\right)+\tilde{\gamma} u^{3}-\frac{\tilde{\delta}}{u},
$$

where $u=u(z)$ and $\tilde{\alpha}, \tilde{\beta}, \tilde{\gamma}, \tilde{\delta}$ are arbitrary constants. By a scaling of the independent variable, the parameters $\tilde{\gamma}$ and $\tilde{\delta}$ can be taken equal to 1 and -1 respectively. There is a transformation between solutions of $\mathrm{P}_{\mathrm{V}}$ and $\mathrm{P}_{\mathrm{III}}$.

Theorem 1.4 ([8, Th. 34.1], [13, $\S 32.7(\mathrm{vi})])$. Let $u=u(z, \tilde{\alpha}, \tilde{\beta})(\tilde{\gamma}=1, \tilde{\delta}=-1)$ be a solution of $P_{\mathrm{III}}$ such that $R=u^{\prime}-\varepsilon u^{2}-(\tilde{\alpha} \varepsilon-1) u / z+1 \neq 0$. Then the function

$$
y(t)=1-\frac{2}{R(\sqrt{2 t})}, \quad \varepsilon^{2}=1
$$

is a solution of $P_{\mathrm{V}}$ with parameters

$$
A=\frac{(\tilde{\beta}-\tilde{\alpha} \varepsilon+2)^{2}}{32}, B=-\frac{(\tilde{\beta}+\tilde{\alpha} \varepsilon-2)^{2}}{32}, C=-\varepsilon, D=0 .
$$

\footnotetext{
${ }^{1}$ http://dlmf.nist.gov/32.7
} 
The inverse transformation is given by the following theorem.

Theorem 1.5 ([8, Th. 34.3]). Let $y(t)$ be a solution of $P_{\mathrm{V}}$ with parameters $A, B, C^{2}=1, D=0$. Then the function

$$
u(z)=\frac{\sqrt{2 t} y}{\Phi(t)}, \quad z^{2}=2 t, \quad \Phi(t)=t y^{\prime}-\sqrt{2 A} y^{2}+(\sqrt{2 A}+\sqrt{-2 B}) y-\sqrt{-2 B} \neq 0
$$

is a solution of $P_{\mathrm{III}}$ with parameters

$$
\tilde{\alpha}=2 C(\sqrt{2 A}-\sqrt{-2 B}-1), \tilde{\beta}=2(\sqrt{2 A}+\sqrt{-2 B}), \tilde{\gamma}=1, \tilde{\delta}=-1 .
$$

\section{MAIN RESUltS}

In this section we relate the recurrence coefficients to the solutions of the fifth and third Painlevé equations and identify the discrete system (6) (7).

2.1. The Toda system. It is well known that for a measure of the form $e^{t x} d \mu(x)$ the recurrence coefficients satisfy

$$
\left\{\begin{array}{l}
\left(a_{n}^{2}\right)^{\prime}:=\frac{d}{d t}\left(a_{n}^{2}\right)=a_{n}^{2}\left(b_{n}-b_{n-1}\right), \\
b_{n}^{\prime}:=\frac{d}{d t} b_{n}=\left(a_{n+1}^{2}-a_{n}^{2}\right),
\end{array}\right.
$$

with initial conditions $a_{n}^{2}(0)$ and $b_{n}(0)$ given by the recurrence coefficients of the orthogonal polynomials for the measure $\mu$ (see, e.g., [10, §2.8], 11]). The weight (41) is of this form when we put $a=e^{t}$. Hence, the recurrence coefficients of the generalized Charlier polynomials on the lattice $\mathbb{N}$, when viewed as functions of the parameter $a$ with respect to weight (44), satisfy

$$
\left\{\begin{array}{l}
\left(a_{n}^{2}\right)^{\prime}:=\frac{d}{d a}\left(a_{n}^{2}\right)=\frac{a_{n}^{2}}{a}\left(b_{n}-b_{n-1}\right), \\
b_{n}^{\prime}:=\frac{d}{d a} b_{n}=\frac{1}{a}\left(a_{n+1}^{2}-a_{n}^{2}\right) .
\end{array}\right.
$$

We get this system from (14) by applying the transformation $a=\exp (t)$ (see [3]). The weight (9) is also of this form when $a=e^{t}$, since

$$
w(k+1-\beta)=\frac{\Gamma(\beta) e^{t(k+1-\beta)}}{\Gamma(2-\beta) k !(2-\beta)_{k}} .
$$

Hence, the recurrence coefficients for the shifted lattice also satisfy the Toda system (15). Also for the bi-lattice we have that $w(x)=e^{t x} \Gamma(\beta) / \Gamma(\beta+x) \Gamma(x+1)$ when $a=e^{t}$, which is of the required form, so that the recurrence coefficients again satisfy the Toda system (15). The only difference between these three cases is in the initial conditions which correspond to the recurrence coefficients of the generalized Charlier polynomials with $n=0$ on respectively the lattice $\mathbb{N}$, the shifted lattice $\mathbb{N}+1-\beta$ and the bi-lattice $\mathbb{N} \cup(\mathbb{N}+1-\beta)$.

2.2. Relation to the Bäcklund transformation of the fifth Painlevé equation. First, let us obtain a nonlinear discrete equation for $b_{n}(a)$. Denoting $a_{n}^{2}=x_{n}$, we have from equation (7) that

$$
x_{n+1}=\frac{a\left(a+n(\beta-n-1)+\left(1+2 n-\beta-b_{n}\right) b_{n}-x_{n}\right)}{a-x_{n}} .
$$


Equation (6) gives

$$
x_{n}=\frac{a n}{\beta-n+b_{n-1}+b_{n}} .
$$

Substituting the last equation and (16) into equation (6) with $n+1$ we have a nonlinear difference equation of the form

$$
F\left(b_{n-1}, b_{n}, b_{n+1}, a\right)=0,
$$

for some $F$ which we do not write out explicitly, since it is quite long. We will not really need its explicit form since it is just an auxiliary equation for the derivation of the continuous Painlevé equation. We shall show later that it can be obtained from the Bäcklund transformation of the fifth Painlevé equation.

To derive the differential equation for $b_{n}(a)$ we use (16) and from equation (6) we have

$$
b_{n-1}=\frac{a n+n x_{n}-\beta x_{n}-b_{n} x_{n}}{x_{n}} .
$$

Substituting this into the first equation of (15) we get

$$
x_{n}^{\prime}=\frac{\left(\beta-n+2 b_{n}\right) x_{n}-a n}{a}
$$

and from the second equation of (15) we have

$$
b_{n}^{\prime}=\frac{a\left(n-a+n^{2}-n \beta\right)-a(1+2 n-\beta) b_{n}+a b_{n}^{2}+2 a x_{n}-x_{n}^{2}}{a\left(x_{n}-a\right)} .
$$

Differentiating equation (18) and using the expression for $x_{n}^{\prime}$, we get an equation for $b_{n}^{\prime \prime}, b_{n}^{\prime}, b_{n}$ and $x_{n}$. Finally, we can eliminate $x_{n}$ by computing the resultant of this equation and equation (18) and we get a nonlinear second-order and second-degree equation

$$
G\left(b_{n}^{\prime \prime}, b_{n}^{\prime}, b_{n}, a\right)=0 .
$$

Now the difficulty is in identifying this equation. Let us scale the independent variable $b_{n}(a)=B_{n}\left(a / k_{1}\right)$ and take $a=k_{1} t$. Equation (19) becomes $G_{1}\left(B_{n}^{\prime \prime}, B_{n}^{\prime}, B_{n}, t\right)$ $=0$, where the differentiation is with respect to $t$. The following result holds.

Theorem 2.1. The equation $G_{1}\left(B_{n}^{\prime \prime}, B_{n}^{\prime}, B_{n}, t\right)=0$ is reduced to the fifth Painlevé equation $P_{\mathrm{V}}$ by the following transformation:

$$
B_{n}(t)=\frac{1+n+(\beta-3 n-2) y+(1+2 n-\beta) y^{2}+t y^{\prime}}{2 y(y-1)},
$$

where $y=y(t)$ satisfies $P_{\mathrm{V}}$ with parameters given by

$$
A=\frac{(\beta-1)^{2}}{2}, \quad B=-\frac{(n+1)^{2}}{2}, C=2 k_{1}, \quad D=0 .
$$

Remark 2. It is known that $\mathrm{P}_{\mathrm{V}}$ with $D=0$ can be reduced to the third Painlevé equation ([8, [13, §32.7(vi)], but this was already known to Gambier in 1910). However, to show that equation (17) can be obtained from the Bäcklund transformation, it is more convenient to use $\mathrm{P}_{\mathrm{V}}$.

Remark 3. The parameters (21) are invariant under $\beta \rightarrow 2-\beta$; compare with the weight on the shifted lattice (9). 
Let $k_{1}=1$ and $a=t$. We use Theorem 1.2 to get $y_{n+1}$ and $y_{n-1}$ from $y=y_{n}$. In particular,

$$
y_{n-1}=1-\frac{4 t(y-1) y^{2}}{(\beta-1)^{2}(y-1)^{2} y^{2}-\left((n+1)(y-1)-t y^{\prime}\right)^{2}}
$$

is a solution of $\mathrm{P}_{\mathrm{V}}$ with $A=(\beta-1)^{2} / 2, B=-n^{2} / 2, C=2, D=0$ and

$$
y_{n+1}=1-\frac{4 t(y-1) y^{2}}{(\beta-1)^{2}(y-1)^{2} y^{2}-\left((n+1)(y-1)+t y^{\prime}\right)^{2}}
$$

is a solution of $\mathrm{P}_{\mathrm{V}}$ with $A=(\beta-1)^{2} / 2, B=-(n+2)^{2} / 2, C=2, D=0$ provided that $y=y(t)$ is a solution of $\mathrm{P}_{\mathrm{V}}$ with (21). Using (20) we can express $b_{n \pm 1}$ in terms of $y^{\prime}, y$. For instance,

$$
b_{n+1}=\frac{1+n+(\beta-3 n-4) y+(3+2 n-\beta) y^{2}-t y^{\prime}}{2 y(y-1)} .
$$

Substituting (20) and the expressions of $b_{n \pm 1}$ into (17), we indeed see that they satisfy the equation. Therefore, the system (6) - (7) is related to the Bäcklund transformation of $\mathrm{P}_{\mathrm{V}}$.

Finally we use Theorem 1.5 to obtain the parameters of $\mathrm{P}_{\text {III }}$. Taking $k_{1}=1 / 2$ from (21) we get the following values for different choices of the square roots:

$$
\begin{gathered}
\tilde{\alpha}=2(1+n-\beta), \tilde{\beta}=-2(n+\beta), \\
\tilde{\alpha}=-2(3+n-\beta), \tilde{\beta}=2(n+\beta), \\
\tilde{\alpha}=2(n-1+\beta), \tilde{\beta}=-2(2+n-\beta), \\
\tilde{\alpha}=-2(1+n+\beta), \tilde{\beta}=2(2+n-\beta) .
\end{gathered}
$$

Taking $k_{1}=1 / 2, \quad a=t / 2$ we change variables $t \rightarrow \sqrt{2 t}=z$ in (19) and take $b_{n}(a)=\tilde{B}_{n}(z)$. Then the functions

$$
\begin{gathered}
\tilde{B}_{n}(z)=-\frac{z+u(2 \beta-2 n-1+z u)+z u^{\prime}}{4 u}, \\
\tilde{B}_{n}(z)=\frac{1}{4}\left(1+2 n-2 \beta+z\left(\frac{8(n+1)}{z-u(z u+2 \beta-2 n-5)-z u^{\prime}}-\frac{u^{\prime}+u^{2}+1}{u}\right)\right), \\
\tilde{B}_{n}(z)=-\frac{z+u(2 \beta-2 n-1+z u)+z u^{\prime}}{4 u}, \\
\tilde{B}_{n}(z)=\frac{1}{4}\left(1+2 n-2 \beta+z\left(\frac{8(n+1)}{z+u(1+2 n+2 \beta-z u)-z u^{\prime}}-\frac{1+u^{2}+u^{\prime}}{u}\right)\right)
\end{gathered}
$$

give the transformations to the third Painlevé equation for the function $u=u(z)$ with parameters (22)- 25) respectively.

In case $\beta=1$ we get from (22)-(25) either

$$
\tilde{\alpha}=2 n, \tilde{\beta}=-2(n+1)
$$

or

$$
\tilde{\alpha}=-2(n+2), \tilde{\beta}=2(n+1) \text {. }
$$


2.3. Initial conditions. In this section we study the initial conditions (8), (10), (11) for the generalized Charlier weight (4) on the lattice $\mathbb{N}$, on the shifted lattice $\mathbb{N}+1-\beta$ and on the bi-lattice respectively. We show that the initial conditions correspond to classical solutions of $\mathrm{P}_{\mathrm{V}}$.

First we get that the equation (19) with $n=0$ has solutions satisfying

$$
a^{2} b_{0}^{\prime}+a b_{0}^{2}-a(1-\beta) b_{0}-a^{2}=0,
$$

where $^{\prime}=d / d a$. The initial conditions (8), (10), (11) satisfy this equation. Moreover, by using the formulas from [1, 13, or computational software, it can be shown that the general solution of (26) is given by (11). We note that the Toda system (15) and the discrete system (6) - (17) are unchanged under the transformation $\beta \rightarrow 2-\beta, a_{n}^{2}=\hat{a}_{n}^{2}, \quad b_{n}=\hat{b}_{n}-1+\beta$. Also equation (26) is unchanged after this transformation with $n=0$.

Using the transformation (20) with $k_{1}=1, a=t$ in (26) we get a first-order second-degree equation

$$
(y-1)\left(1+y\left((\beta-1)^{2} y^{2}-\left(4 t+(\beta-1)^{2}\right) y-1\right)\right)+2 t(y-1) y^{\prime}-t^{2} y^{\prime 2}=0,
$$

for which the solutions satisfy $\mathrm{P}_{\mathrm{V}}$ with parameters $A=(\beta-1)^{2} / 2, B=-1 / 2, C=$ $2, D=0$. Using the transformation $y \rightarrow 1 / y$ in $\mathrm{P}_{\mathrm{V}}$ we get equation (38.7) in 8 ] for special values of the parameters. Hence, the solutions of $\mathrm{P}_{\mathrm{V}}$ in terms of classical functions correspond to the initial conditions for the recurrence coefficients.

Remark 4. The recurrence coefficients $a_{n}^{2}$ and $b_{n}$ can always be written [4] as ratios of Hankel determinants containing the moments of the orthogonality measure. However, the explicit determinant formulas of classical solutions for all the Painlevé equations are known.

2.4. Revisiting case $\beta=1$. It is shown in $[3$ that the monic polynomials orthogonal with respect to a semi-classical variation of the Charlier weight given by

$$
w(k)=\frac{a^{k}}{(k !)^{2}}, \quad a>0, \quad k \in \mathbb{N},
$$

have recurrence coefficients which satisfy $\mathrm{P}_{\mathrm{V}}$ with

$$
A=-B=\frac{n^{2}}{8}, \quad C=0, \quad D=-8 .
$$

This weight can be obtained from weight (4) by taking $\beta=1$. However, if we take the parameter $\beta=1$ in (21), we get $\mathrm{P}_{\mathrm{V}}$ with

$$
A=0, \quad B=-\frac{(n+1)^{2}}{2}, C=2 k_{1}, \quad D=0,
$$

which seems to be a contradiction. In the following we show how to reduce (19) to $\mathrm{P}_{\mathrm{V}}$ with parameters (27).

The following transformation exists between solutions of $\mathrm{P}_{\mathrm{V}}$ [ . Let $y=y(z)$ be a solution of $\mathrm{P}_{\mathrm{V}}$ with $A=-B=\alpha / 4, C=0, D=4 \gamma$. Then the function

$$
\tilde{y}(t)=\frac{(y+1)^{2}}{4 y}, t=z^{2}
$$


is a solution of $\mathrm{P}_{\mathrm{V}}$ with $A=\alpha, B=0, C=\gamma, D=0$. So, if we start with a solution $y$ of $\mathrm{P}_{\mathrm{V}}$ with parameters (28), we can first use the transformation $y \rightarrow 1 / y$ to get the parameters

$$
A=\frac{(n+1)^{2}}{2}, \quad B=0, \quad C=-2 k_{1}, \quad D=0,
$$

then the transformation (29) to get solutions with

$$
A=\frac{(n+1)^{2}}{8}, \quad B=-\frac{(n+1)^{2}}{8}, C=0, \quad D=-8 k_{1} .
$$

Then taking $k_{1}=1$ and using Theorem 1.3 (e.g., a transformation from the example in case $\varepsilon=1$ ) we get a solution of $\mathrm{P}_{\mathrm{V}}$ with parameters (27). Explicitly this gives

$$
b_{n}(t)=\frac{n+7 n y^{2}-n y^{3}-2 z y^{\prime}-y\left(7 n+2 z y^{\prime}\right)}{8 y(y-1)}, \quad t=z^{2},
$$

where $y=y(z)$ is a solution of $\mathrm{P}_{\mathrm{V}}$ with (27). Equation (26) is also transformed to a first-order second-degree equation for which the solutions satisfy $\mathrm{P}_{\mathrm{V}}$. Hence, we get that the solutions of $\mathrm{P}_{\mathrm{V}}$ in terms of classical special functions generate the initial conditions for the system (6)-(7).

\section{Discussion}

The recurrence coefficients for semi-classical weights are often related to the solutions of discrete and continuous Painlevé equations (e.g., [5, 6] and see also the overview in [3). The known results and our recent findings of the relations between the recurrence coefficients of the (generalized) Charlier and Meixner polynomials and the fifth Painlevé equation (12) with parameters $(A, B, C, D)$ can be summarized as follows. We assume below that $k \in \mathbb{N}=\{0,1, \ldots\}$.

(1) (a) The weight $a^{k} / k$ !, $a>0$, is a classical Charlier weight and the recurrence coefficients can be computed explicitly.

(b) It is shown in [3] that the recurrence coefficients for the weight $a^{k} /(k !)^{2}$, $a>0$, are related to the solutions of the fifth Painlevé equation $\mathrm{P}_{\mathrm{V}}$ with parameters $\left(n^{2} / 8,-n^{2} / 8,0,-8\right)$. Moreover, as shown in this paper, by using certain transformations, they are also related to solutions of $\mathrm{P}_{\mathrm{V}}$ with parameters $\left(0,-(n+1)^{2} / 2,2,0\right)$ (this is the special case of $1(\mathrm{c}))$.

(c) As shown in this paper, the recurrence coefficients for the weight $a^{k} /\left((\beta)_{k} k !\right), \quad a>0, \beta \neq 1$, are related to solutions of $\mathrm{P}_{\mathrm{V}}$ with $\left((\beta-1)^{2} / 2,-(n+1)^{2} / 2,2,0\right)$.

(2) (a) The weight $(\beta)_{k} c^{k} / k$ !, $\quad \beta>0,0<c<1$, is the classical Meixner weight, and the recurrence coefficients are known explicitly.

(b) It is shown in [3] that the recurrence coefficients for $(\beta)_{k} c^{k} /(k !)^{2}$, $\beta>0, c>0$, are related to solutions of $\mathrm{P}_{\mathrm{V}}$ with $\left((\beta-1)^{2} / 2\right.$, $\left.-(\beta+n)^{2} / 2,2 n,-2\right)$.

(c) It is shown in [6] that the recurrence coefficients for $(\gamma)_{k} c^{k} /\left(k !(\beta)_{k}\right)$, $c, \beta, \gamma>0$, are related to solutions of $\mathrm{P}_{\mathrm{V}}$ with $\left((\gamma-1)^{2} / 2,-(\gamma-\right.$ $\left.\left.\beta+n)^{2} / 2, k_{1}(\beta+n),-k_{1}^{2} / 2\right)\right), k_{1} \neq 0$. The case $\gamma=1$ corresponds to a classical Charlier weight on the shifted lattice $\mathbb{N}+1-\beta$. The case $\beta=\gamma$ gives the Charlier weight and the case $\beta=1$ is given in $2(\mathrm{~b})$. 
Therefore, we have essentially two different cases for $\mathrm{P}_{\mathrm{V}}$ : $D=0$ for the recurrence coefficients of the generalizations of the Charlier weight and $D \neq 0$ for the recurrence coefficients of the generalizations of the Meixner weight. Note that the case $D=0$ can be reduced to the third Painlevé equation.

It is interesting to study weights on shifted lattices. For instance, there is no shifted lattice for the weight $a^{k} /(k !)^{2}$ since both lattices coincide. Since the lattices are determined by the zeros of the weight, one could have more than one lattice if one takes $a^{k} /\left((\beta)_{k}(\gamma)_{k}(\delta)_{k} k\right.$ !), which is zero for $k=-1, k=-\beta, k=-\gamma$ and $k=-\delta$.

It is an interesting open problem to study recurrence coefficients for more complicated weights such as $a^{k} /(k !)^{3}$ or $(\gamma)_{k}(\beta)_{k} c^{k} /(k !)^{2}$ and others. The Toda system will be the same but the discrete system will be much more complicated (maybe higherorder discrete Painlevé equations will be needed). Will this lead to the Painlevé equations (with more general parameters $A, B, C, D$ depending on the parameters in the weight) or some higher-order equations in some hierarchy of the Painlevé equations? The direct calculations will be cumbersome and other techniques such as using the Lax pairs or others will be needed to tackle such problems.

\section{REFERENCES}

[1] M. Abramowitz and I. Stegun, Handbook of Mathematical Functions, Dover Publications, New York, 1992. MR.1225604 (94b:00012)

[2] V.E. Adler, Nonlinear chains and Painlevé equations, Physica D 73 (1994), 335-351. MR 1280883 (95c:58157)

[3] L. Boelen, G. Filipuk and W. Van Assche, Recurrence coefficients of generalized Meixner polynomials and Painlevé equations, J. Phys. A: Math. Theor. 44 (2011), 035202 (19 pp). $\operatorname{MR} 2749070(2011 \mathrm{j}: 33036)$

[4] T. S. Chihara, An Introduction to Orthogonal Polynomials, Gordon and Breach, New York, 1978. MR0481884(58:1979)

[5] G. Filipuk, W. Van Assche, and L. Zhang The recurrence coefficients of semi-classical Laguerre polynomials and the fourth Painlevé equation, arXiv:1105.5229v1 [math.CA]

[6] G. Filipuk and W. Van Assche, Recurrence coefficients of a new generalization of the Meixner polynomials, SIGMA Symmetry Integrability Geom. Methods Appl. 7 (2011), 068, 11 pages.

[7] A.S. Fokas and M.J. Ablowitz, On a unified approach to transformations and elementary solutions of Painlevé equations, J. Math. Phys. 23 (1982), 2033-2042. MR679998(84b:34004)

[8] V.I. Gromak, I. Laine and S. Shimomura, Painlevé Differential Equations in the Complex Plane, Vol. 28, Studies in Mathematics, de Gruyter, Berlin, New York, 2002. MR.1960811 (2003m:34210)

[9] M.N. Hounkonnou, C. Hounga, and A. Ronveaux, Discrete semi-classical orthogonal polynomials: generalized Charlier, J. Comput. Appl. Math. 114 (2000), 361-366. MR 1737084 (2001m:33010)

[10] M.E.H. Ismail, Classical and Quantum Orthogonal Polynomials in One Variable, Encyclopedia of Mathematics and its Applications 98, Cambridge University Press, 2005. MR2191786 (2007f:33001)

[11] J. Moser, Finitely many mass points on the line under the influence of an exponential potential — an integrable system, Dynamical Systems, Theory and Applications. Lecture Notes in Physics 38, Springer, Berlin, 1975, pp. 467-497. MR0455038 (56:13279)

[12] M. Noumi, Painlevé equations through symmetry, Translations of Mathematical Monographs, 223, American Mathematical Society, Providence, RI, 2004. MR.2044201(2005b:34183)

[13] F.W.J. Olver, D.W. Lozier, R.F. Boisvert, and C.W. Clark, NIST Handbook of Mathematical Functions, NIST and Cambridge University Press, 2010. MR2723248(2012a:33001)

[14] C. Smet and W. Van Assche, Orthogonal polynomials on a bi-lattice, Constr. Approx., DOI:10.1007/S00365-011-9145-8, arXiv:1101.1817v1 [math.CA]

[15] T. Tokihiro, B. Grammaticos, and A. Ramani, From the continuous $P_{\mathrm{V}}$ to discrete Painlevé equations, J. Phys. A: Math. Gen. 35 (2002), 5943-5950. MR1930545 (2003j:39049) 
[16] V.V. Tsegelnik, The Painlevé type equations: analytical properties of solutions and their applications, Habilitation thesis, Minsk, 2001 (in Russian).

[17] W. Van Assche and M. Foupouagnigni, Analysis of non-linear recurrence relations for the recurrence coefficients of generalized Charlier polynomials, J. Nonlinear Math. Phys. 10 (2003), suppl. 2, 231-237. MR2063533 (2005f:33020)

Faculty of Mathematics, Informatics and Mechanics, University of Warsaw, Banacha 2, WARSAW 02-097, Poland

E-mail address: filipuk@mimuw.edu.pl

Department of Mathematics, Ku Leuven, Celestijnenlaan 200B, Box 2400, BE-3001 Leuven, Belgium

E-mail address: Walter.VanAssche@wis.kuleuven.be 